\title{
Experimental and Computational Vibration Study of Amino Acids
}

\author{
Musa E. Mohamed ${ }^{1, *}$, Abdelhafeez M. A. Mohammed ${ }^{2}$ \\ ${ }^{1}$ Department of Chemistry, College of Applied and Industrial Sciences, University of Bahri, Sudan \\ ${ }^{2}$ Department of Chemistry, Faculty of Education, Alzaiem Alazhari University, Sudan \\ *E-mail address: musa.elballa@gmail.com
}

\begin{abstract}
Vibrational studies of amino acids experimentally and theoretically have been performed. The Semi-empirical methods optimization by PM6 and RM1 on the $l$ - and $d$-amino acids (alanine, phenylalanine, aspartic and glutamic acid), showed no difference in energy between $l$-and $d$-isomers. The vibrational frequencies were calculated by semi-emprical methods (PM6 and RM1) and ab initio methods (B3LYP/6-31+G(d) and were scaled down by factors of 0.925 (RM1), 1.09 (PM6) and 0.89 $(\mathrm{B} 3 \mathrm{LYP} / 6-31+\mathrm{G}(\mathrm{d}))$. The calculated and experimental vibrational frequencies have shown good general agreement.
\end{abstract}

Keywords: Vibrational studies; Amino Acids; semi-empirical methods (PM6 and RM1); ab initio methods (B3LYP/6-31+G(d)

\section{INTRODUCTION}

Amino acids are the molecular building blocks of peptides and proteins. The unit structure adopted by amino acids, on condensation into larger molecules, effectively determines their secondary structure in crystalline samples.

Amino acid molecules have a zwitterionic structure when they are in the liquid or solid phase. Indeed these two cases allow hydrogen bonds to be formed, stabilizing the ionic conformation $\mathrm{R}-\mathrm{CH}\left(\mathrm{COO}^{-}\right) \mathrm{NH}^{3+}$. On the Contrary, the intermolecular bonds are not present in the gas phase and these molecules have a non-zwitterionic structure $\mathrm{R}-\mathrm{CH}(\mathrm{COOH}) \mathrm{NH}_{2}$. This has been confirmed by experimental studies of the amino acids glycine [1-3] and alanine [4]. There are two long-standing problems encountered in the study of zwitterionic amino acids by infrared (IR) absorption spectroscopy and $a b$ initio molecular orbital methods. The first concerns the inability of standard ab initio molecular orbital calculations (for isolated molecules) to provide optimized structures for the monomeric zwitterionic forms of the amino acids. A number of molecular orbital studies (self-consistent field (SCF) methods) [5-7] have shown that the zwitterionic amino acids do not exist in the gas phase, as isolated monomers.

This conclusion has been confirmed experimentally by matrix isolation (MI) studies [810], a technique in which the amino acids are first vaporized, then trapped and isolated in inert gas matrices at very low temperatures. The MI infrared spectra clearly show that amino 
acids are present in un-ionized neutral forms after evaporation. For the monomers, the structure optimizations started at the zwitterionic forms, with large basis sets, always converge to the un-ionized neutral molecules. For some smaller basis sets (e.g. 6-31G(d)), convergence to structures with strong intramolecular hydrogen bonding is obtained. These are not expected to be real structures but occur as a consequence of the relatively low theoretical level of the calculations.

The second problem concerns the measurement of IR absorption spectra of monomers of the amino acids in their zwitterionic forms. In the gas phase the amino acids occur in their un-ionized forms. The zwitterions have to be stabilized by their local environment. This is achieved in polar solutions and in the solid state. Measurement of the IR absorption spectra of the solid ( $\mathrm{KBr}$ pellet), and solution are not of the monomer but of tiny crystals in the case of $\mathrm{KBr}$ pellets, and of strong solute-solvent hydrogen bonding in the case of aqueous solutions.

Thus, with the existing standard sampling techniques IR absorption spectra can only be measured when the zwitterions are strongly perturbed, either by neighbouring zwitterions (crystals in $\mathrm{KBr}$ pellets), or in solution (strong $\mathrm{H}$-bonding). Other traditional methods involving evaporation of sample (such as MI, molecular beam and gas phase) to obtain monomeric spectra are essentially inapplicable to studying zwitterionic amino acids because of the structural transformation, from zwitterionic to neutral un-ionized form.

To carry out meaningful calculations on the zwitterionic forms of the amino acids, either solvation effects or specific intermolecular interactions must be included. Calculations including specific intermolecular interactions are usually impracticable, because of the number of molecules (large multimers or clusters) that must be included in the computation. However, the inclusion of a general solvation effect in the calculations can be readily undertaken. Self-consistent reaction field (SCRF) methods [11], which are implemented in Gaussian packages include the solvation effect.<smiles>[NH3+]C(Cc1ccccc1)C(=O)[O-]</smiles>

Phenylalanine<smiles>[NH3+]C(CC(=O)O)C(=O)[O-]</smiles>

Aspartic Acid<smiles>CC([NH3+])C(=O)[O-]</smiles>

Alanine<smiles>[NH3+]C(CCC(=O)O)C(=O)[O-]</smiles>

Glutamic Acid

Figure 1. Zwitterionic Structures of Amino Acids.

These methods model the solvent as a continuum of uniform dielectric constant $(\varepsilon)$, the reaction field, which can interact with the solute molecules and lead to net stabilization. It should be noted that the solvent used in SCRF calculations is restricted to non-aqueous 
system [11], because as pointed out by Foresman, where intermolecular interactions are relatively large the continuum model is inappropriate, and specific interactions have also to be included. For aqueous solutions this would require the inclusion of a shell of water molecules inside the cavity in addition to the solute [12]. It was established for glycine in aqueous solution, two water molecules are sufficient to stabilize the glycine zwitterions [13].

Therefore, the use of the continuum model in which no specific intermolecular interactions are included in the calculations excludes comparison with the spectra of aqueous solution and crystal. Moreover, the IR and Raman spectra of amino acids in aqueous solution are usually broad, overlapped or incomplete as a result of strong solvent absorption and solute-solvent interaction. Even in the event that appropriately calculated spectra could be obtained, satisfactory and complete comparisons would be difficult to establish.

Motivated by the need to solve the above problems of (i) measuring the absorption spectra of monomers of zwitterions of amino acids and (ii) undertaking appropriate $a b$ initio calculations; Jensen, J. H. and Gordon, M. S. have developed and optimized new IR sampling technique, and have carried out non-aqueous SCRF molecular orbital calculations. The IR absorption spectra of glycine [14] and alanine [15] have previously been measured with this technique, and the SCRF calculated spectra for these zwitterions were shown to be in good agreement with the measured spectra.

$L$-alanine is the smallest naturally occurring chiral amino acid. The assignment of its fundamental vibrations is of importance in modeling amino acids and the nature of the mechanism of their inversion.

The vibrational spectra of $\alpha$ - and $\beta$-alanine molecules in both zwitterionic and neutral forms are studied by FT-IR, Raman and MI-IR spectroscopy with the aid of results from theoretical SCF-MO ab initio calculations. The spectroscopic data obtained under the various experimental conditions (crystalline phase; low temperature matrix isolated molecules) made it possible to undertake a detailed assignment of the vibration frequencies [16].

The infrared spectra of two conformers of the nonionized alanine have been analyzed and assigned using DFT/B3LYP/aug-cc-pVDZ and MP2/aug-cc-pVDZ for geometry and frequency theoretical calculations. These methods yields vibrational frequencies in excellent agreement with experimental data [17].

The infrared spectra of the alanine molecule also have been studied in solid as well as in aqueous solution [18]. The vibration frequencies for the fundamental modes of alanine in neutral and zwitterionic form have been calculated using AM1, RHF, and DFT method with different basis sets [18]. RHF/6-31G, DFT/6-31G, 6-31+G* and 6-311++G** calculations for vibrational frequencies of both $l$ - and $d$-alanine and zwitterionic alanine (zala) have been performed in both gas phase and in aqueous solution [18].

It was concluded that while there is no significant difference between the corresponding frequencies of $l$ - and $d$-alanine in gas phase, the situation is not the same for zala and alanine in water [18]. A solvation model (PCM) for neutral alanine and zala at DFT/6-31+G* and 6$311++\mathrm{G}^{* *}$ level has also been performed. Gas phase and salvation using Polarized Continuum (PCM) model calculations for alanine and zala reveal that neutral alanine is more stable in gas phase while the reverse is true in aqueous medium. A comparison between the experimentally observed IR spectra of alanine in solid and water solution does not show much variation in corresponding frequencies but theoretically some differences are predicted.

The infrared spectra of $l$-aspartic acid, $l$-aspartic-d4 acid, and $l$-aspartic $-{ }^{15} \mathrm{~N}$ acid as solid samples [19] data was used to propose a general assignment of the fundamental modes in the basis of the isotopic shifts measured. 
The infrared spectrum and molecular structure of zwitterionic $l-\beta$-phenylalanine were studied by means of matrix isolation method and ab initio molecular orbital calculation. The self-consistent reaction field calculations at HF/6- $(311 \mathrm{G}(\mathrm{d}, \mathrm{p}))$ level were carried out on zwitterionic phenylalanine present in a continuum of $\mathrm{KBr}$. Good agreement in the terms of both frequencies and intensities was found between the calculated and observed full mid-IR spectra [20].

The Fourier transform infrared spectra of the grown $l$-phenylalanine crystals were recorded in the frequency region $450 \mathrm{~cm}^{-1}-4000 \mathrm{~cm}^{-1}$ [21], the recorded FTIR spectra was compared with the standard spectra of the functional groups. The presence of all the functional groups occurring in $l$-phenylalanine was confirmed.

The MNDO-scaled harmonic force field of glutamic acid was obtained in the space of a set of non-redundant local-symmetrized internal coordinates [22] by a set of scaling factors transferred from aspartic acid. The theoretical vibration frequencies for the fundamentals were successfully compared with the observed values, and the frequencies of the ${ }^{15} \mathrm{~N}$ and $d_{4}$ isotopic derivatives were also calculated and compared with the experiments [22]. These results and the description of the normal modes followed by means of the potential energy distribution, agree with the previous assignments proposed for most of the observed bands, showing an extensive coupling among the bending and skeletal stretching coordinates [22].

The molecular structure of glutamic acid in the non-zwitterionic form has been optimized by using the semiempirical (MNDO, AM1) methods and ab initio calculation at the 4-31G level [23]. The results were compared with previous reported data for other amino acids. The $a b$ initio optimized structure was used as the starting point for a further force field calculation, and vibration frequencies and theoretical assignments were thus obtained. In order to compare these results with experiments, the FT-IR spectrum of glutamic acid in an argon matrix was recorded and the measured frequencies were successfully compared with theoretical values, which were previously scaled by using a set of scaling factors [23].

According to the reasons mentioned above in the introduction, most molecular orbital calculations on amino acids are performed on the un-ionized species. In this work we calculated the IR spectra of our amino acids in the non-zwitterionic form using semiempirical and $a b$ initio force field calculations, and compared the results with experimental spectra.

\section{METHODS OF CALCULATION}

\section{1. Semiemprical Methods}

The Semi empirical computational methods RM1 and PM6 were used as implemented in MOPAC2007 package, on a personal computer. In this procedure, we first searched for the energy minima on the potential energy surface of the selected amino acids ( $l$-alanine, $l$ phenylalanine, $l$-aspartic and $l$-glutamic acid). The molecular structures corresponding to the global minima in the potential energy surface for the non-zwitterionic amino acids were predicted in the four cases starting from a structure with standard parameters, which were optimized simultaneously employing very restricted convergence criteria in the last steps of the computations.

The optimized RM1 and PM6 geometries were used as the reference geometry to calculate the vibrational frequencies of the amino acids ( $l$-alanine, $l$-phenylalanine, $l$-aspartic and $l$-glutamic acid). It is possible to find a local minimum of the potential energy surface of the amino acids, where all the calculated frequencies were positive, thus allowing the comparison with experimental results. A scaling factor of 0.925 for RM1 common to all 
frequencies is used to adjust the theoretical frequencies before it was compared with experimental [24], and 1.09 factor was used for PM6.

\section{2. Ab initio Methods}

The ab initio molecular orbital calculations were carried out employing the Gaussian 03 program. In this procedure we first searched for the energy minima on the potential energy surface of the amino acids corresponding to the lowest energy conformer and then calculated infrared frequencies using harmonic approximations. Initially, geometry was optimized using Hartee-Fock level of theory with $6-31+\mathrm{G}(\mathrm{d})$ basis set $(\mathrm{RHF} / 6-31+\mathrm{G}(\mathrm{d}))$ and less effective methods of Density Functional Theory (DFT) at B3LYP/6-31+G(d) which consider electron correlations with three-parameters hybrid functions combined with the Lee,Yang and ParrCorrelation function [25]. The optimized geometry of amino acids ( $l$-alanine, $l$-phenylalanine, $l$-aspartic and $l$-glutamic acid) was used to calculate the vibrational frequencies. The calculated frequencies values were scaled down by a single factor of 0.89 before it were compared with experimental data.

\section{EXPERIMENTAL}

The FTIR spectra of $l$ - amino acids (alanine, phenylalanine, aspartic and glutamic acid) were recorded with the Fourier Transform Infrared spectrometer model $8400 \mathrm{~S}$, in the frequency region $400-4000 \mathrm{~cm}^{-1}$. Samples in the solid states were measured in $\mathrm{KBr}$ matrix. Pellets were obtained with a hydraulic press.

\section{RESULTS AND DISCUSSION}

Taking into account the geometries of $l$ - and $d$-amino acids (alanine, phenylalanine, aspartic and glutamic acid), no difference in energy minima was observed for the two isomers by semiempirical methods (RM1 and PM6), see Table (1)

The optimized semiempirical RM1, PM6 and ab initio B3LYP/6-31+G(d) geometries were used as starting point to carry force field calculations for non-zwitterionic, $l$-alanine, $l$ phenylalanine, $l$-aspartic and $l$-glutamic acid. To minimize the systematic errors of these calculations, scaling factors of $0.89,0.925$ and 1.09 were applied for ab initio, RM1 and PM6 respectively. In Tables 2-5, we have listed the scaled calculated vibrational frequencies of non-zwitterionic, $l$-alanine, $l$-phenylalanine, $l$-aspartic and $l$-glutamic acid respectively. We have also proposed assignments for the observed frequencies.

\section{1. Assignment of $l$-alanine}

The non-zwitterionic structure of $l$-alanine is shown in Figure 2. The assignment for calculated vibrational frequencies and those observed in the IR spectra are shown in Table 2. The calculated and experimental vibrational frequencies show a good general agreement. The frequencies observed at $3099 \mathrm{~cm}^{-1}$ is assigned to the asymmetric $\mathrm{NH}$ stretching mode. 
Table 1. Relative Stability of Amino Acids ( $\Delta \mathrm{E})$ at Different Semiempirical Methods RM1 and PM6.

\begin{tabular}{lcc}
\hline \multicolumn{3}{c}{ Heat of formation in Kcal/mol } \\
\hline Amino acids & PM6 & RM1 \\
$l$-alanine & -92.7728 & -98.13294 \\
$d$-alanine & -92.7728 & -98.13295 \\
$l$-phenylalanine & -69.7187 & -73.25132 \\
$d$-phenylalanine & -71.9725 & -75.59622 \\
$l$-aspartic & -184.2430 & -189.38191 \\
$d$-aspartic & -184.2430 & -189.38191 \\
$l$-glutamic & -185.8963 & -187.89090 \\
$d$-glutamic & -185.8963 & -187.89090 \\
\hline
\end{tabular}

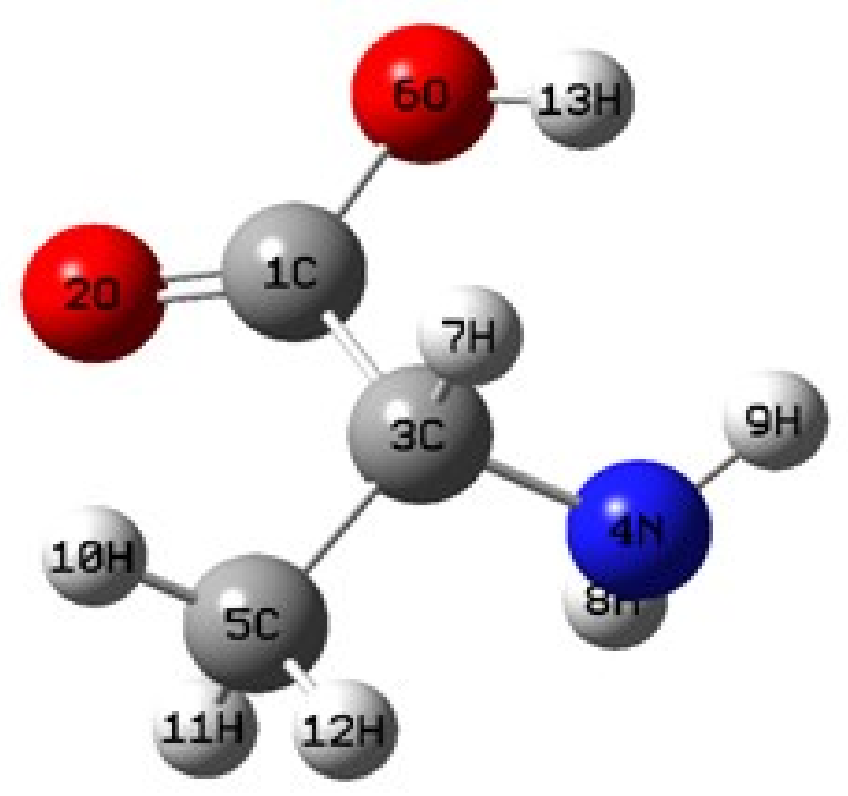

Figure 2. Molecular Structure of $l$-Alanine as Predicted by the $a b$ initio Method, with the Atomic Numbering Used in This Work.

The corresponding theoretical values at B3LYP/6-31+G(d), RM1 and the PM6 level are $3310 \mathrm{~cm}^{-1}, 3085 \mathrm{~cm}^{-1}$ and $3082 \mathrm{~cm}^{-1}$, respectively, literature work at $3389 \mathrm{~cm}^{-1}$ (ab initio [18]), $3085 \mathrm{~cm}^{-1}$ (AM1 [18]), which shows semi-empirical methods give closed value to the experimental methods than ab initio.

The peak calculated at $3167 \mathrm{~cm}^{-1}, 3071 \mathrm{~cm}^{-1}$ and $3041 \mathrm{~cm}^{-1}$ having no counterpart in the IR spectrum refers to the $\mathrm{CH}$ stretching mode. The peak observed at $2926 \mathrm{~cm}^{-1}$ in the IR is 
attributed to the asymmetric $\mathrm{CH}$ stretching in the $\mathrm{CH}_{3}$ group, since the corresponding theoretical values are $3096 \mathrm{~cm}^{-1}, 3065 \mathrm{~cm}^{-1}$ and $3035 \mathrm{~cm}^{-1}$ for ab initio, RM1 and PM6 respectively, in the literature $2898 \mathrm{~cm}^{-1}$ by ab initio [18] and $2758 \mathrm{~cm}^{-1}$ for Am1 [18].

The peak observed at $2604 \mathrm{~cm}^{-1}$ in the IR spectrum is assigned to the NH stretching in the $\mathrm{NH}_{3}$ group. The corresponding calculated frequencies are $2691 \mathrm{~cm}^{-1}, 2633 \mathrm{~cm}^{-1}$ and 2748 $\mathrm{cm}^{-1}$ by ab initio, RM1 and PM6 repectively. The frequency of $2631 \mathrm{~cm}^{-1}$ was calculated by ab initio [18].

The calculated peaks at $1643 \mathrm{~cm}^{-1}, 1809 \mathrm{~cm}^{-1}$ and $1979 \mathrm{~cm}^{-1}$ for the asym. $\mathrm{COO}^{-}$ stretching coupled with a NH bending mode, does not appear in the IR spectrum. Barthes et. al. [26] have reported this frequency at $1630 \mathrm{~cm}^{-1}$ in IR spectrum. The peak at $1595 \mathrm{~cm}^{-1}$ in the IR spectrum is assigned to the $\mathrm{NH}_{2}$ scissoring mode, according to calculated values at $1522 \mathrm{~cm}^{-1}, 1514 \mathrm{~cm}^{-1}$ and $1755 \mathrm{~cm}^{-1}$.

The calculation of this peak was reported at $1620 \mathrm{~cm}^{-1}$ (ab initio [18]) and $1464 \mathrm{~cm}^{-1}$ (Am1 [18]). Other vibrations in the finger print region $\left(400-1354 \mathrm{~cm}^{-1}\right)$ and the corresponding calculated frequencies by ab initio, RM1 and PM6 are listed in Table 2.

Table 2. Calculated Vibrational Frequencies $\mathrm{cm}^{-1}$ for $l$-Alanine Compared with Experimental.

\begin{tabular}{cccccl}
\hline Mode & Obs. & RM1 & PM6 & Ab initio & Assignment \\
\hline $\mathbf{v}_{\mathbf{1}}$ & 3099 & 3085 & 3082 & 3310 & Asym. $\mathrm{NH}_{2}$ stretching \\
$\mathbf{v}_{\mathbf{2}}$ & & 3071 & 3041 & 3167 & $\mathrm{CH}$ stretching in $\mathrm{CH}_{3}$ \\
$\mathbf{v}_{\mathbf{3}}$ & 2926 & 3065 & 3035 & 3096 & Asm.CH stretching in $\mathrm{CH}_{3}$ \\
$\mathbf{v}_{\mathbf{4}}$ & & 2853 & 2936 & 2796 & Puckering of $\mathrm{CH}_{3}$ \\
$\mathbf{v}_{\mathbf{5}}$ & & 2801 & 2924 & 2774 & $\mathrm{CH}$ stretching \\
$\mathbf{v}_{\mathbf{6}}$ & & 2798 & 2903 & 2717 & $\mathrm{CH}$ strething in $\mathrm{CH}_{3}$ group \\
$\mathbf{v}_{\mathbf{7}}$ & 2604 & 2633 & 2748 & 2691 & $\mathrm{NH}$ stretchingin $\mathrm{NH}_{2}$ \\
$\mathbf{v}_{\mathbf{8}}$ & & 1809 & 1979 & 1643 & $\mathrm{Asym} . \mathrm{COO}-$ stretching $+\mathrm{NH}$ bend. \\
$\mathbf{v}_{\mathbf{9}}$ & 1595 & 1514 & 1755 & 1522 & $\mathrm{NH}_{2}$ bending \\
$\mathbf{v}_{\mathbf{1 0}}$ & 1354 & 1410 & 1475 & 1354 & $\mathrm{NH}_{2}$ scissoring \\
$\mathbf{v}_{\mathbf{1 1}}$ & 1307 & 1256 & 1328 & 1343 & $\mathrm{NH}_{3}$ puckering \\
$\mathbf{v}_{\mathbf{1 2}}$ & & 1245 & 1285 & 1276 & $\mathrm{CH}$ deformation \\
$\mathbf{v}_{\mathbf{1 3}}$ & & 1226 & 1248 & 1262 & $\mathrm{CH}$ deformation \\
$\mathbf{v}_{\mathbf{1 4}}$ & & 1198 & 1246 & 1195 & $\mathrm{CH}$ puckering \\
$\mathbf{v}_{\mathbf{1 5}}$ & & 1194 & 1230 & 1150 & $\mathrm{Mixed}$ vibration $\left(\mathrm{NH}_{2}\right.$ puck $+\mathrm{CH}_{3}$ def \\
$\mathbf{v}_{\mathbf{1 6}}$ & & 1182 & 1184 & 1133 & $\mathrm{CH}$ bend. $+\mathrm{NH}$ bend. \\
$\mathbf{v}_{\mathbf{1 7}}$ & 1149 & 1104 & 1176 & 1044 & $\mathrm{CH}_{2}$ bend. $+\mathrm{CH}$ bend. $+\mathrm{NH}_{2}$ bend \\
$\mathbf{v}_{\mathbf{1 8}}$ & 1114 & 1062 & 1165 & 1005 & $\mathrm{C}-\mathrm{C}$ stretch $+\mathrm{CH}$ bend. $+\mathrm{NH}_{\text {bend. }}$ \\
$\mathbf{v}_{\mathbf{1 9}}$ & 1014 & 985 & 1073 & 966 & $\mathrm{H}-\mathrm{N}-\mathrm{C}-\mathrm{C}$ bend. \\
\hline & & & & & \\
\hline
\end{tabular}




\begin{tabular}{llllll}
\hline $\mathbf{v}_{\mathbf{2 0}}$ & & 965 & 984 & 913 & $\mathrm{H}-\mathrm{C}-\mathrm{C}-\mathrm{H}$ bend. $+\mathrm{NH}_{2}$ def. \\
$\mathbf{v}_{\mathbf{2 1}}$ & 918 & 930 & 935 & 821 & $\mathrm{CNH}$ bend \\
$\mathbf{v}_{\mathbf{2 2}}$ & 850 & 826 & 893 & 816 & $\mathrm{CN}$ stretch. $+\mathrm{C}-\mathrm{C}$ stretching \\
$\mathbf{v}_{\mathbf{2 3}}$ & 769 & 677 & 840 & 706 & $\mathrm{HNC}+\mathrm{CCH}$ bending \\
$\mathbf{v}_{\mathbf{2 4}}$ & 646 & 657 & 676 & 654 & $\mathrm{CCC}+\mathrm{COO}$ bending \\
$\mathbf{v}_{\mathbf{2 5}}$ & 543 & 570 & 558 & 569 & $\mathrm{OCOH}+\mathrm{NH}_{2}$ bending \\
$\mathbf{v}_{\mathbf{2 6}}$ & 412 & 486 & 472 & 441 & $\mathrm{OH}$ bending \\
$\mathbf{v}_{\mathbf{2 7}}$ & & 378 & 426 & 432 & $\mathrm{NCC}$ bending $+\mathrm{CCCO}$ \\
\hline
\end{tabular}

\section{2. Assignment of $l$-Phenylalanine}

The Non-zwitterionic structure of $l$-phenly-alanine is shown in Figure 3. The assignment for calculated frequencies and those observed in the IR spectra are shown in Table 2. The characteristic bands at $3436 \mathrm{~cm}^{-1}$ due to $\mathrm{NH}$ asymmetric stretching, confirmed the existence of amino group, the corresponding calculated IR spectrum are $3187 \mathrm{~cm}^{-1}, 3083 \mathrm{~cm}^{-1}$ and $3084 \mathrm{~cm}^{-1}$ at B3LYP/6-31+G(d), RM1 and PM6 respectively, the same peak at $3150 \mathrm{~cm}^{-1}$ was predicted by SCRF calculation [20] and assigned to $\mathrm{NH}$ asymmetric stretching frequency.

The peak calculated at $3106 \mathrm{~cm}^{-1}, 3065 \mathrm{~cm}^{-1}$ and $3040 \mathrm{~cm}^{-1}$ not appearing in the experimental IR spectrum are for the NH symmetric stretching, the peak may have disappeared because of $\mathrm{H}$-bonding.

The band at $3028 \mathrm{~cm}^{-1}$ establishes the presence of $\mathrm{CH}_{2}$ asymmetric stretching, as the corresponding theoretical values of this work at $3030 \mathrm{~cm}^{-1}, 3009 \mathrm{~cm}^{-1}$ and $3016 \mathrm{~cm}^{-1}$, and calculated by SCRF at $3001 \mathrm{~cm}^{-1}$ [20] are very near.

The peak at $2918 \mathrm{~cm}^{-1}$ is assigned to $\mathrm{CH}$ stretching in the phenyl ring, since the corresponding calculated values are at $2854 \mathrm{~cm}^{-1}, 2847 \mathrm{~cm}^{-1}$ and $3006 \mathrm{~cm}^{-1}$ by B3LYP/6$31+\mathrm{G}(\mathrm{d}), \mathrm{RM} 1$ and PM6 respectively, the calculation of this band in the literature is $2996 \mathrm{~cm}^{-}$ ${ }^{1}$ by ab initio (SCRF) [20].

The calculated values at $2843 \mathrm{~cm}^{-1}, 2835 \mathrm{~cm}^{-1}, 2821 \mathrm{~cm}^{-1}, 2818 \mathrm{~cm}^{-1}$ at B3LYP/6$31+\mathrm{G}(\mathrm{d}), 2836 \mathrm{~cm}^{-1}, 2831 \mathrm{~cm}^{-1}, 2824 \mathrm{~cm}^{-1}, 2821 \mathrm{~cm}^{-1}$ at RM1, and $3002 \mathrm{~cm}^{-1}, 2997 \mathrm{~cm}^{-1}$, $2992 \mathrm{~cm}^{-1}, 2983 \mathrm{~cm}^{-1}$ at PM6 also are for the $\mathrm{CH}$ stretchings in phenyl ring which are not observed in IR experimental spectrum.

The bands calculated at $2759 \mathrm{~cm}^{-1}, 2776 \mathrm{~cm}^{-1}$ and $2907 \mathrm{~cm}^{-1}$ are assigned to $\mathrm{CH}$ stretching, since the corresponding band in the literature calculated by ab initio (SCRF) [20] is at $2930 \mathrm{~cm}^{-1}$.

The peaks calculated at $2723 \mathrm{~cm}^{-1}, 2706 \mathrm{~cm}^{-1}$ by B3LYP/6-31+G(d), at $2717 \mathrm{~cm}^{-1}, 2628$ $\mathrm{cm}^{-1}$ by RM1 and at $2889 \mathrm{~cm}^{-1}, 2711 \mathrm{~cm}^{-1}$ by PM6 for the asymmetric $\mathrm{CH}_{2}$ stretching and symmetric $\mathrm{CH}_{2}$ stretching respectively, are not experimentally observed.

The band observed at $1683 \mathrm{~cm}^{-1}$ is assigned to $\mathrm{COO}^{-}$asymmetric stretching, since the corresponding theoretical values are $1637 \mathrm{~cm}^{-1}, 1809 \mathrm{~cm}^{-1}$ and $1688 \mathrm{~cm}^{-1}$ by ab initio, RM1 and PM6 respectively. Assignments of other vibrations from $1600-300 \mathrm{~cm}^{-1}$ are listed in Table 3. 


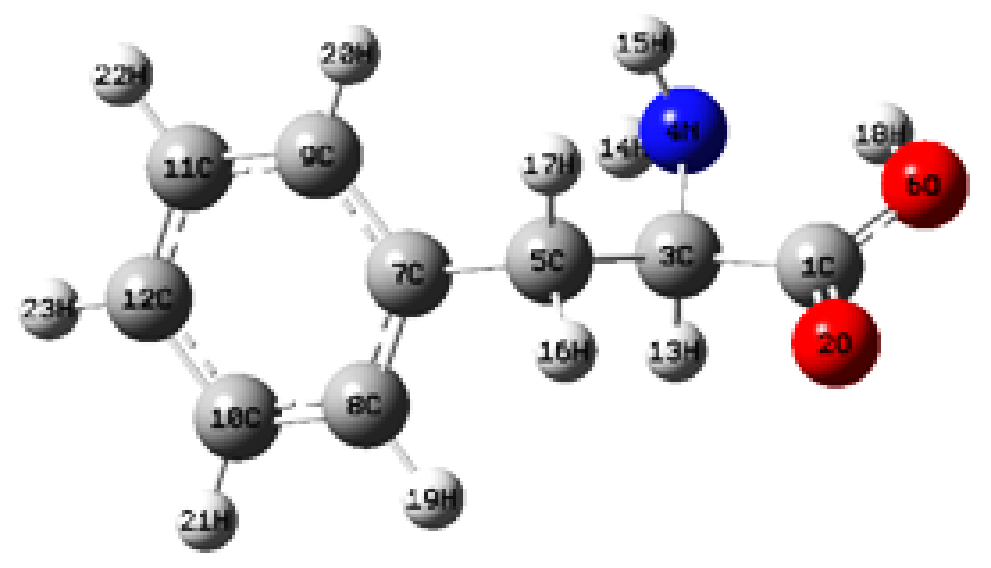

Figure 3. Molecular Structure of $l$-Phenylalanine.

Table 3. Calculated Vibrational Frequencies in $\mathrm{cm}^{-1}$ for $l$-Phenylalanine Compared with Experimental.

\begin{tabular}{ccccll}
\hline Mode & Obs. & RM1 & PM6 & Ab initio & Assignment \\
\hline$v_{1}$ & 3436 & 3083 & 3084 & 3187 & NH asymmetric stretching \\
$v_{2}$ & & 3065 & 3040 & 3106 & NH symmetric stretching \\
$v_{3}$ & 3028 & 3009 & 3016 & 3030 & $\mathrm{CH}_{2}$ asymmetric stretch. \\
$v_{4}$ & 2918 & 2847 & 3006 & 2854 & $\mathrm{CH}$ stretching in phenyl Ring \\
$v_{5}$ & & 2836 & 3002 & 2843 & $\mathrm{CH}$ stretching in phenyl Ring \\
$v_{6}$ & & 2831 & 2997 & 2835 & $\mathrm{CH}$ stretching in phenyl Ring \\
$v_{7}$ & & 2824 & 2992 & 2821 & $\mathrm{CH}$ stretching in phenyl Ring \\
$v_{8}$ & & 2821 & 2983 & 2818 & $\mathrm{CH}$ stretching in phenyl Ring \\
$v_{9}$ & & 2776 & 2907 & 2759 & $\mathrm{CH}^{2}$ stretching \\
$v_{10}$ & & 2717 & 2889 & 2723 & $\mathrm{CH}_{2}$ asymmetric stretching \\
$v_{11}$ & & 2628 & 2711 & 2706 & $\mathrm{CH}_{2}$ symmetric stretching \\
$v_{12}$ & 1683 & 1809 & 1688 & 1637 & $\mathrm{COO}^{-}$asymmetric stretching \\
$v_{13}$ & 1596 & 1607 & 1539 & 1510 & $\mathrm{NH}$ asymmetric bending \\
$v_{14}$ & 1478 & 1601 & 1534 & 1472 & $\mathrm{NH}$ asymmetric bending \\
$v_{15}$ & 1449 & 1508 & 1500 & 1454 & Phenyl ring Stretching \\
$v_{16}$ & 1393 & 1457 & 1422 & 1369 & Phenyl ring Stretching \\
$v_{17}$ & 1358 & 1418 & 1384 & 1343 & Phenyl ring Stretching \\
$v_{18}$ & 1332 & 1401 & 1343 & 1329 & $\mathrm{CH}_{2}$ bending \\
$v_{19}$ & & 1282 & 1238 & 1267 & $\mathrm{NH}_{\text {symmetric bending }}$ \\
\hline & & & & & \\
\hline
\end{tabular}




\begin{tabular}{|c|c|c|c|c|c|}
\hline$v_{20}$ & & 1239 & 1216 & 1251 & NH symmetric bending \\
\hline$v_{21}$ & & 1231 & 1208 & 1227 & $\mathrm{COO}^{-}$symmetric stretching \\
\hline$v_{22}$ & 1218 & 1222 & 1191 & 1213 & $\mathrm{CH}_{2}$ bending \\
\hline$v_{23}$ & & 1190 & 1170 & 1201 & $\mathrm{CH}$ bending in phenyl ring \\
\hline$v_{24}$ & 1161 & 1174 & 1168 & 1157 & C-C stretching \\
\hline$v_{25}$ & 1130 & 1154 & 1155 & 1119 & $\mathrm{CH}_{2}$ bending \\
\hline$v_{26}$ & 1097 & 1142 & 1144 & 1095 & Phenyl ring Stretching \\
\hline$v_{27}$ & 1081 & 1125 & 1133 & 1084 & $\mathrm{CH}$ bending in phenyl ring \\
\hline$v_{28}$ & & 1109 & 1122 & 1078 & $\mathrm{C}-\mathrm{C}$ stretching in phenyl ring \\
\hline$v_{29}$ & 1040 & 1091 & 1090 & 1059 & $\mathrm{CH}$ bending in phenyl ring \\
\hline$v_{30}$ & & 1058 & 1079 & 1019 & $\mathrm{CH}$ bending in phenyl ring \\
\hline$v_{31}$ & & 1048 & 1061 & 995 & $\mathrm{NH}$ rocking \\
\hline$v_{32}$ & & 1041 & 1052 & 980 & $\mathrm{CH}$ rocking in phenyl ring \\
\hline$v_{33}$ & & 1011 & 1035 & 938 & Out-of- plane $\mathrm{CH}$ stretching in phenyl \\
\hline$v_{34}$ & & 993 & 984 & 924 & NH rocking \\
\hline$v_{35}$ & & 970 & 965 & 903 & $\mathrm{CH}$ bending in phenyl ring \\
\hline$v_{36}$ & & 925 & 941 & 892 & Out-of- plane $\mathrm{CH}$ stretching in phenyl \\
\hline$v_{37}$ & 879 & 907 & 922 & 871 & Out-of- plane $\mathrm{CH}$ stretching in phenyl \\
\hline$v_{38}$ & 859 & 885 & 909 & 835 & Phenyl ring Stretching \\
\hline$v_{39}$ & & 881 & 875 & 827 & Out-of- plane $\mathrm{CH}$ stretching in phenyl \\
\hline$v_{40}$ & & 850 & 853 & 792 & $\mathrm{CH}_{2}$ rocking \\
\hline$v_{41}$ & & 814 & 834 & 776 & $\mathrm{C}-\mathrm{COO}^{-}$stretching \\
\hline$v_{42}$ & & 804 & 823 & 770 & Out-of- plane $\mathrm{CH}$ stretching in phenyl \\
\hline$v_{43}$ & 744 & 742 & 791 & 763 & $\mathrm{CH}_{2}$ rocking \\
\hline$v_{44}$ & 703 & 737 & 742 & 725 & C-N stretching \\
\hline$v_{45}$ & & 666 & 681 & 677 & Out-of- plane $\mathrm{CH}$ stretching in phenyl \\
\hline$v_{46}$ & 642 & 613 & 647 & 645 & $\mathrm{COO}^{-}$bending \\
\hline$v_{47}$ & & 575 & 597 & 632 & Out-of -plane ring deformation \\
\hline$v_{48}$ & 579 & 555 & 573 & 564 & $\mathrm{COO}^{-}$waging \\
\hline$v_{49}$ & & 529 & 557 & 549 & In plane ring deformation \\
\hline$v_{50}$ & 480 & 472 & 529 & 510 & In-plane ring deformation \\
\hline$v_{51}$ & & 415 & 483 & 463 & $\mathrm{COO}^{-}$rocking \\
\hline
\end{tabular}




\begin{tabular}{lllll}
\hline$v_{52}$ & 412 & 429 & 432 & Out-of-plane ring deformation \\
$v_{53}$ & 396 & 412 & 370 & Out-of-plane ring deformation \\
$v_{54}$ & 329 & 350 & 369 & C-C deformation \\
$v_{55}$ & 319 & 328 & 315 & C-N deformation \\
$v_{56}$ & 298 & 296 & 299 & C-C deformation \\
$v_{57}$ & 233 & 255 & 258 & N-H deformation \\
\hline
\end{tabular}

\section{3. Assignment of $l$-Aspartic Acid}

The Non-zwitterionic structure of $l$-aspartic acid is shown in Figure 4. The calculated frequencies and the observed in the IR spectra are shown in Table 4. The calculated and experimental vibrational frequencies show a reasonable general agreement. The characteristic bands at $3420 \mathrm{~cm}^{-1}$ and $3250 \mathrm{~cm}^{-1}$ due to $\mathrm{OH}$ stretching, have the corresponding calculated IR spectrum at $3280 \mathrm{~cm}^{-1}, 3275 \mathrm{~cm}^{-1}$ at B3LYP/6-31+G(d), $3090 \mathrm{~cm}^{-1}, 3079 \mathrm{~cm}^{-1}$ at RM1, and $3058 \mathrm{~cm}^{-1}, 3018 \mathrm{~cm}^{-1}$ at PM6 respectively.

The calculated IR spectra of $\mathrm{OH}$ stretching in the literature is at $3481 \mathrm{~cm}^{-1}$ by B3lyp/3$21 \mathrm{G}$ [27]. The peak appearing at $3141 \mathrm{~cm}^{-1}, 3011 \mathrm{~cm}^{-1}$ in observed IR spectrum is assigned to the NH asymmetric and symmetric stretching, since the corresponding calculated IR spectrum are $3189 \mathrm{~cm}^{-1}, 3114 \mathrm{~cm}^{-1}$ at B3LYP/6-31+G(d), $3017 \mathrm{~cm}^{-1}, 3016 \mathrm{~cm}^{-1}$ at RM1, and $2966 \mathrm{~cm}^{-1}$, $2887 \mathrm{~cm}^{-1}$ at PM6 respectively. The corresponding spectrum of $\mathrm{NH}$ in the literature is 3400 $\mathrm{cm}^{-1}$ by B3lyp/3-21G [27].

The bands at $2942 \mathrm{~cm}^{-1}, 2853 \mathrm{~cm}^{-1}$ establish the presence of $\mathrm{CH}$ asymmetric stretching in the $\mathrm{CH}_{2}$, as the corresponding theoretical values at $2775 \mathrm{~cm}^{-1}, 2738 \mathrm{~cm}^{-1}$ at B3LYP/6$31+\mathrm{G}(\mathrm{d}), 2764 \mathrm{~cm}^{-1}, 2709 \mathrm{~cm}^{-1}$ at RM1, and $2865 \mathrm{~cm}^{-1}, 2771 \mathrm{~cm}^{-1}$ at PM6, and in the literature the peak assigned at $3103 \mathrm{~cm}^{-1}$ by ab initio [27]. The peak at $1620 \mathrm{~cm}^{-1}$ is assigned to $\mathrm{C}=\mathrm{O}$ asymmetric stretching, the corresponding calculated values are at $1607 \mathrm{~cm}^{-1}, 1826$ and $1821 \mathrm{~cm}^{-1}$ respectively. In the literature $\mathrm{C}=\mathrm{O}$ asymmetric stretching is calculated at 1764 $\mathrm{cm}^{-1}$ by ab initio [27].

Table 4. Calculated Vibrational Frequencies in $\mathrm{cm}^{-1}$ for $l$-Aspartic Acid Compared with Experimental.

\begin{tabular}{llllll}
\hline Mode & Obs. & RM1 & PM6 & Ab initio & Assignment \\
\hline $\mathbf{v}_{\mathbf{1}}$ & 3420 & 3090 & 3058 & 3280 & OH stretching \\
$\mathbf{v}_{\mathbf{2}}$ & 3250 & 3079 & 3018 & 3275 & OH stretching \\
$\mathbf{v}_{\mathbf{3}}$ & 3141 & 3017 & 2966 & 3189 & $\mathrm{NH}$ asymmetric stretching \\
$\mathbf{v}_{\mathbf{4}}$ & 3011 & 3016 & 2887 & 3114 & $\mathrm{NH}$ symmetric stretching \\
$\mathbf{v}_{\mathbf{5}}$ & 2942 & 2764 & 2865 & 2775 & $\mathrm{CH}$ stretching in $\mathrm{CH}_{2}$ \\
$\mathbf{v}_{\mathbf{6}}$ & 2853 & 2709 & 2771 & 2738 & $\mathrm{CH}$ stretching in $\mathrm{CH}_{2}$ \\
$\mathbf{v}_{\mathbf{7}}$ & & 2605 & 2767 & 2623 & $\mathrm{CH}$ stretching in $\mathrm{CH}_{2}$ \\
$\mathbf{v}_{\mathbf{8}}$ & 1692 & 1831 & 1832 & 1619 & $\mathrm{C}=\mathrm{O}$ stretching $+\mathrm{NHN}$ bending \\
\hline
\end{tabular}




\begin{tabular}{|c|c|c|c|c|c|}
\hline$v_{9}$ & 1620 & 1826 & 1821 & 1607 & $\mathrm{C}=\mathrm{O}$ asymmetric stretching \\
\hline$v_{10}$ & 1538 & 1528 & 1500 & 1504 & $\mathrm{NHN}$ bending $+\mathrm{C}=\mathrm{O}$ stretching \\
\hline$v_{11}$ & 1398 & 1417 & 1480 & 1305 & $\mathrm{CH}$ bending $+\mathrm{HCH}$ bending \\
\hline$v_{12}$ & 1377 & 1399 & 1336 & 1293 & $\mathrm{CH}$ bending \\
\hline$v_{13}$ & 1292 & 1262 & 1298 & 1243 & $\mathrm{HCH}$ bending $+\mathrm{CC}$ stretching $+\mathrm{CN}$ str. \\
\hline$v_{14}$ & 1213 & 1241 & 1267 & 1217 & $\mathrm{C}-\mathrm{C}$ stretching $+\mathrm{C}-\mathrm{N}$ stretching \\
\hline$v_{15}$ & & 1233 & 1242 & 1181 & $\mathrm{NH}_{2}$ bending $+\mathrm{CN}$ stretching \\
\hline$v_{16}$ & 1142 & 1214 & 1202 & 1149 & $\mathrm{C}-\mathrm{OH}$ symmetric stretching in $\mathrm{COOH}$ \\
\hline$v_{17}$ & 1117 & 1186 & 1188 & 1132 & $\mathrm{C}-\mathrm{OH}$ asymmetric stretch. in $\mathrm{COOH}$ \\
\hline$v_{18}$ & 1073 & 1164 & 1178 & 1082 & $\mathrm{CH}_{2}$ rocking $+\mathrm{C}-\mathrm{C}$ stretching \\
\hline$v_{19}$ & & 1108 & 1131 & 1050 & $\mathrm{C}-\mathrm{CH}_{2}-\mathrm{C}$ bending \\
\hline $\mathbf{v}_{20}$ & & 1082 & 1110 & 1029 & C-C stretching \\
\hline$v_{21}$ & 987 & 1055 & 1022 & 1011 & $\mathrm{C}-\mathrm{C}$ stretching $+\mathrm{C}-\mathrm{N}$ stretching \\
\hline$v_{22}$ & 895 & 1017 & 1011 & 937 & $\mathrm{NH}_{2}$ wagging \\
\hline $\mathbf{v}_{\mathbf{2 3}}$ & 854 & 947 & 978 & 859 & $\mathrm{C}-\mathrm{C}$ stretching $+\mathrm{CO}$ stretching $+\mathrm{NH}_{2}$ bend. \\
\hline $\mathbf{v}_{24}$ & & 918 & 920 & 803 & C-C-C wagging \\
\hline$v_{25}$ & & 865 & 861 & 788 & C-C-C bending \\
\hline$v_{26}$ & & 680 & 846 & 761 & OH rocking \\
\hline$v_{27}$ & 693 & 635 & 637 & 654 & $\mathrm{OH}$ rocking \\
\hline $\mathbf{v}_{28}$ & 642 & 589 & 572 & 597 & $\mathrm{OH}$ rocking \\
\hline$v_{29}$ & 580 & 535 & 542 & 578 & COO- bending \\
\hline$v_{30}$ & 525 & 523 & 516 & 546 & COO- bending \\
\hline$v_{31}$ & & 499 & 492 & 511 & $\mathrm{OH}$ wagging $+\mathrm{CH}_{2}$ wagging \\
\hline$v_{32}$ & 478 & 466 & 474 & 474 & $\mathrm{NH}_{2}$ twisting \\
\hline $\mathbf{v}_{33}$ & & 456 & 440 & 461 & $\mathrm{NH}_{2}-\mathrm{C}-\mathrm{COOH}$ bending \\
\hline$v_{34}$ & 397 & 355 & 347 & 361 & $\mathrm{NH}_{2}$ wagging \\
\hline$v_{35}$ & & 325 & 317 & 320 & Torsion $\mathrm{COO}^{-}$ \\
\hline$v_{36}$ & 294 & 306 & 287 & 295 & Torsion $\mathrm{COO}^{-}$ \\
\hline
\end{tabular}

The band at $1538 \mathrm{~cm}^{-1}$ is assigned to NHN bending $+\mathrm{C}=\mathrm{O}$ stretching, calculated values at $1504 \mathrm{~cm}^{-1}, 1528 \mathrm{~cm}^{-1}, 1500 \mathrm{~cm}^{-1}$ at B3LYP/6-31+G(d), RM1 and PM6 respectively. Assignments of other vibrations are listed in Table 4. 


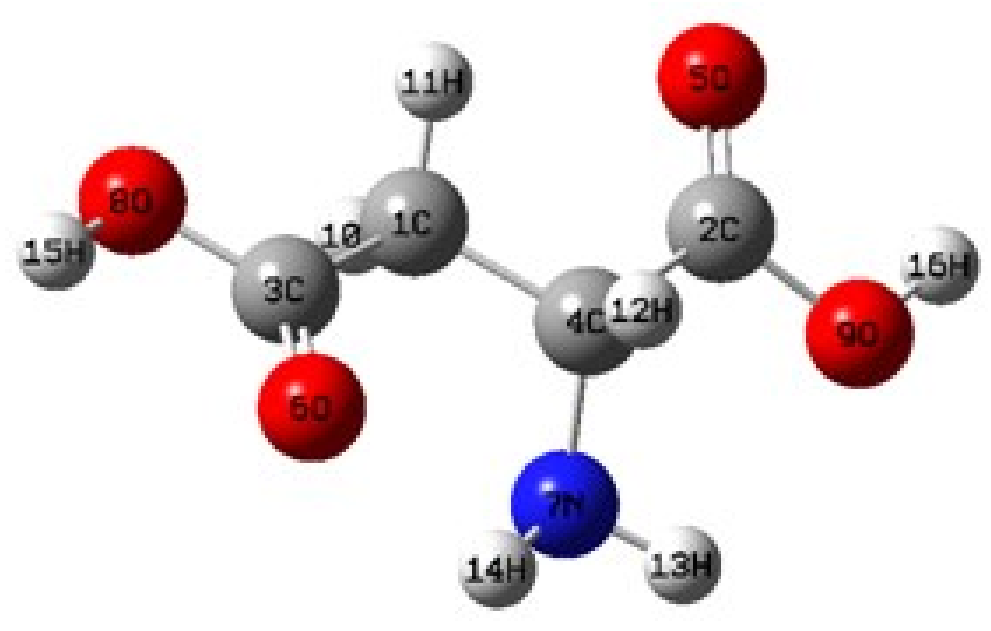

Figure 4. Molecular Structure of $l$-Aspartic Acid.

\section{4. Assignment of $l$-Glutamic Acid}

The Non-zwitterionic structure of $l$-glutamic acid is shown in Figure 5. The calculated frequencies and those observed in the IR spectra are shown in Table 5. There is a little increase in frequency value in the high-frequencies region between $2700-2900 \mathrm{~cm}^{-1}$, between experimental and calculated by ab initio and RM1 but in the case of PM6 it is reasonable, i.e the $\mathrm{CH}_{2}$ symmetric and asymmetric stretching frequencies bands observed at $2974 \mathrm{~cm}^{-1}, 2965$ $\mathrm{cm}^{-1}$ and $2935 \mathrm{~cm}^{-1}$, while the corresponding calculated frequencies by ab initio and semiempirical RM1 methods are $2770 \mathrm{~cm}^{-1}, 2758 \mathrm{~cm}^{-1}, 2736 \mathrm{~cm}^{-1}$ and $2774 \mathrm{~cm}^{-1}, 2763 \mathrm{~cm}^{-1}$, $2710 \mathrm{~cm}^{-1}$ respectively.

The same calculated peaks by PM6 are at $2904 \mathrm{~cm}^{-1}, 2884 \mathrm{~cm}^{-1}$ and $2876 \mathrm{~cm}^{-1}$. The calculated $\mathrm{CH}_{2}$ spectra in the literature by ab initio $4-31 \mathrm{G}$ is $2972 \mathrm{~cm}^{-1}$ [23]. The peak observed at $1781 \mathrm{~cm}^{-1}$ is assigned to $\mathrm{C}=\mathrm{O}$ stretching frequency, the calculated frequencies are $1610 \mathrm{~cm}^{-1}, 1820 \mathrm{~cm}^{-1}$ and $1806 \mathrm{~cm}^{-1}$ by ab initio, RM1 and PM6. The calculated one in the literature is assigned at $1765 \mathrm{~cm}^{-1}$ by ab initio 4-31G [23]. There is also rise in the frequency value in IR bands in the case of semiempirical RM1 and PM6 methods in frequencies region $700-1000 \mathrm{~cm}^{-1}$ when compared with experimental ones. The other assignments are show in Table 5. The only explanation of the departure of experimental values from calculated values is a possible intramolecular hydrogen bonding forming a cyclic structure, a situation not considered in theory.

Table 5. Calculated Vibrational Frequencies in $\mathrm{cm}^{-1}$ for $l$-Glutamic Acid Compared with Experimental.

\begin{tabular}{llllll}
\hline Mode & Obs. & RM1 & PM6 & Ab initio & Assignment \\
\hline $\mathbf{v}_{\mathbf{1}}$ & & 3101 & 3077 & 3268 & OH stretching \\
$\mathbf{v}_{\mathbf{2}}$ & & 3093 & 3044 & 3170 & OH stretching \\
$\mathbf{v}_{\mathbf{3}}$ & 3020 & 3004 & 2978 & 3130 & $\mathrm{NH}$ asymmetric stretching \\
$\mathbf{v}_{\mathbf{4}}$ & 2994 & 2886 & 2975 & 3089 & $\mathrm{CH}$ stretching in $\mathrm{CH}_{2}$ \\
\hline
\end{tabular}




\begin{tabular}{|c|c|c|c|c|c|}
\hline $\mathbf{v}_{5}$ & 2974 & 2774 & 2904 & 2770 & $\mathrm{CH}_{2}$ asymmetric stretching \\
\hline$v_{6}$ & 2965 & 2763 & 2884 & 2758 & $\mathrm{CH}_{2}$ symmetric stretching \\
\hline$v_{7}$ & 2935 & 2710 & 2876 & 2736 & $\mathrm{CH}_{2}$ symmetric stretching \\
\hline $\mathbf{v}_{8}$ & & 2706 & 2767 & 2721 & $\mathrm{NHN}$ bending $+\mathrm{C}=\mathrm{O}$ stretching \\
\hline$v_{9}$ & & 2621 & 2618 & 2709 & NHN in-plane bending \\
\hline$v_{10}$ & 1781 & 1820 & 1806 & 1610 & $\mathrm{C}=\mathrm{O}$ stretching \\
\hline$v_{11}$ & 1635 & 1799 & 1783 & 1589 & $\mathrm{CH}$ bending $+\mathrm{HCH}$ bending \\
\hline$v_{12}$ & 1405 & 1511 & 1502 & 1512 & $\mathrm{CH}$ in-plane bending \\
\hline$v_{13}$ & 1386 & 1429 & 1348 & 1398 & $\mathrm{CO}$ stretching $+\mathrm{CH}_{2}$ torsion \\
\hline$v_{14}$ & 1337 & 1413 & 1340 & 1319 & $\mathrm{C}-\mathrm{C}$ stretching $+\mathrm{CO}$ stretching \\
\hline$v_{15}$ & 1301 & 1266 & 1328 & 1277 & $\mathrm{CH}_{2}$ torsion \\
\hline$v_{16}$ & 1279 & 1243 & 1281 & 1263 & $\mathrm{CH}_{2}$ torsion $+\mathrm{NH}_{2}$ in-plane bending \\
\hline$v_{17}$ & & 1235 & 1269 & 1236 & $\mathrm{C}-\mathrm{OH}$ asymmetric stretch. in $\mathrm{COOH}$ \\
\hline$v_{18}$ & 1234 & 1218 & 1248 & 1221 & $\mathrm{CN}$ stretching \\
\hline$v_{19}$ & & 1195 & 1237 & 1197 & $\mathrm{C}-\mathrm{CH}_{2}-\mathrm{C}$ bending \\
\hline $\mathbf{v}_{20}$ & & 1190 & 1223 & 1170 & $\mathrm{C}-\mathrm{C}$ stretching \\
\hline$v_{21}$ & 1139 & 1177 & 1130 & 1138 & $\mathrm{OH}$ bending $+\mathrm{CH}_{2}$ torsion $+\mathrm{CN}$ stretching \\
\hline$v_{22}$ & 1124 & 1165 & 1120 & 1125 & $\mathrm{OH}$ in-plane bending \\
\hline$v_{23}$ & 1108 & 1131 & 1097 & 1091 & $\mathrm{CN}$ stretching $+\mathrm{OH}$ in-plane bending \\
\hline$v_{24}$ & 1092 & 1063 & 1091 & 1060 & C-C stretching \\
\hline$v_{25}$ & 1024 & 1089 & 1066 & 1043 & $\mathrm{C}-\mathrm{C}$ stretching $+\mathrm{CH}_{2}$ rocking $+\mathrm{CO}$ stretch. \\
\hline$v_{26}$ & & 1072 & 1052 & 997 & $\mathrm{CC}$ stretching $+\mathrm{NH}_{2}$ in-plane bending \\
\hline$v_{27}$ & & 1012 & 1031 & 946 & $\mathrm{CH}_{2}$ rocking $+\mathrm{CC}$ stretching \\
\hline$v_{28}$ & & 994 & 991 & 920 & $\mathrm{OH}$ rocking \\
\hline $\mathbf{v}_{29}$ & 886 & 951 & 932 & 889 & CC stretching + CO stretching \\
\hline$v_{30}$ & 814 & 877 & 908 & 826 & HOCC out-of-plane bending $+\mathrm{CH}_{2}$ rock. \\
\hline $\mathbf{v}_{31}$ & & 867 & 874 & 803 & $\mathrm{OH}$ wagging $+\mathrm{CH}_{2}$ wagging \\
\hline$v_{32}$ & 754 & 790 & 837 & 752 & $\mathrm{CH}_{2}$ rocking \\
\hline$v_{33}$ & & 662 & 740 & 705 & $\mathrm{NH}_{2}-\mathrm{C}-\mathrm{COOH}$ bending \\
\hline$v_{34}$ & 668 & 590 & 660 & 689 & HOCC out-of-plane bending \\
\hline$v_{35}$ & 646 & 577 & 607 & 640 & $\mathrm{NH}_{2}$ out-of-plane bending \\
\hline$v_{36}$ & 612 & 559 & 598 & 607 & $\mathrm{COOH}$ in-plane bending \\
\hline
\end{tabular}




\begin{tabular}{|c|c|c|c|c|c|}
\hline $\mathbf{v}_{37}$ & 570 & 528 & 577 & 568 & OH out-of-plane bending \\
\hline$v_{38}$ & 553 & 522 & 538 & 510 & OH out-of-plane bending \\
\hline$v_{39}$ & & 481 & 505 & 496 & $\mathrm{COOH}$ in-plane bending \\
\hline$v_{40}$ & & 459 & 499 & 471 & $\mathrm{COOH}$ in-plane bending \\
\hline$v_{41}$ & & 409 & 452 & 420 & $\mathrm{CCC}$ in-plane bending $+\mathrm{CNN}$ in-plane ben \\
\hline$v_{42}$ & & 331 & 337 & 322 & CCC in-plane bending \\
\hline $\mathbf{v}_{43}$ & & 320 & 277 & 275 & $\mathrm{CCN}$ in-plane bendin \\
\hline$v_{44}$ & & 282 & 266 & 265 & $\mathrm{NH}_{2}$ torsion \\
\hline$v_{45}$ & & 235 & 238 & 248 & $\mathrm{CCC}$ in-plane bending \\
\hline
\end{tabular}

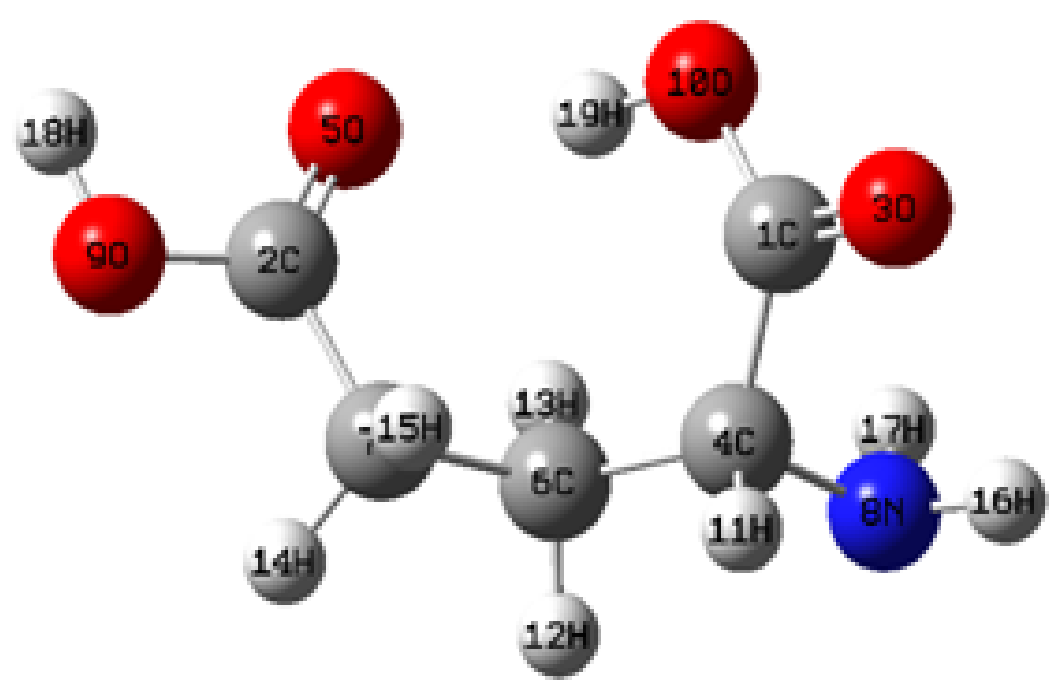

Figure 5. Molecular Structure of $l$-Glutamic Acid.

\section{CONCLUSION}

Assignments of amino acids experimentally and theoretically have been performed. New parameters of semi-empirical methods in MOPAC2007 MP6 and RM1 are used. Comparison of scaled theoretical and experimental vibrationa frequencies exhibit good correlation confirming the reliability of the method employed here.

\section{SUPPLEMENTARY INFORMATIONS}

The supplementary informations (output results of semi-empirical and $a b$ initio methods calculations) are available as free of charge on request. 


\section{References}

[1] Suenram, R. D., Lovas F. J., J. Mol. Spectrosc. 72 (1978) 372.

[2] Suenram, R. D., Lovas F. G., J. Am. Chem. Soc. 102 (1980) 7180.

[3] Iijima K., Tanaka K., Onuma S., J. Mol. Struct. 246 (1991) 257.

[4] Iijima K., Beagley B., J. Mol. Struct. 248 (1991) 133.

[5] Ding Y., Krogh-Jespersen K., Chem. Phys. Lett. 199 (1992) 261.

[6] Sambrano J. R., de Sousa A. R., Queralt J. J., Andrès J., Longo E., Chem. Phys. Lett. 294 (1998) 1.

[7] Császár A. G., J. Phys. Chem. 100 (1996) 3541.

[8] Reva I. D., Plokhotnichenko A. M., Stepanian S. G., Ivanov A. Y., Radchenko E. D., Sheina G. G., Blagoi Y. P., Chem. Phys. Lett. 232 (1995) 141.

[9] Rosado M. T. S., Duarte M. L. R. S., Fausto R., J. Mol. Struct. 410(1997) 343.

[10] Reva I. D., Plokhotnichenko A. M., Stepanian S. G., Ivanov A. Y., Radchenko E. D., Sheina G. G., Blagoi Y. P., J. Mol. Struct. 318 (1994) 1.

[11] Foreman J. B., Frisch Æ., Exploring Chemistry with Electronic Stucture Methods, Second Edition, Gaussian, Pittsburgh, PA, (1996) 237.

[12] Foresman J. B., Private communication, November 3 (1996).

[13] Jensen J. H., Gordon M. S., J. Am. Chem. Soc. 117 (1995) 8159.

[14] Cao X., Fischer G., in: Proceedings of the 22nd Annual Meeting of Australian Society for Biophysics, The Australian National University, Canberra, December, 1998.

[15] Cao X., Fischer G., Spectrochim. Acta Part A 55 (1999) 2329-2342.

[16] Rosado M. T. S., Duarte M. L. R. S., Fausto R., J. Mol. Struct. 410/411 (1997) 343-348.

[17] Stepanian S. G., Reva I. D., Radchenko E. D., Adamowicz L., J. Phys. Chem. A 102 (1998) 4623-4629.

[18] Santosh K., Amareshwar K. R., Rai S. B., Rai D. K., Singh A. N., Singh V. B., J. Mol. Struct. 791 (2006) 23-25.

[19] Lopez N. J. T., Hemandez V., Ramirez F. J., Biopolymers 34 (2004)(8) 1065-1077.

[20] Cao X., Fischer G., J. Mol. Struct. 519 (2000) 153-163.

[21] Mahalakshmi R., Jesuraja S. X., Terome D. S., Cryst.Res. Technol. 41(8) (2006) 780-783.

[22] Ramirez F. J., Lopez J. T. N., Spectrochimica Acta. A 51(2) (1995) 293-302.

[23] Lopez Navarrete J. T., Bencivenni L., Ramondo F., Hernandez V., Ramiraz F. J., J. Mol. Struct. 330 (1995) 261-266.

[24] Ochterski J. W., Vibrational Analysis in Gaussian, Gaussian Inc. Pittshurg, PA, 2000.

[25] Lee C., Yang W., Parr R. G., Phys. Rev. B 37 (1988) 785. 
[26] Barthes M., Vik A. F., Spire A., Bordallo H. N., Eckert J., J. Phys. Chem. A 106 (2002) 5230.

[27] Otavio V. C., Claudio A. T. S., Tiago G., Judith, F., Spectrochimica Acta. A 61(2) (2005) 337-345. 